\title{
Anatomical variations of the stylopharyngeus and superior constrictors in relation to their function
}

\author{
Da-Yae Choi ${ }^{1}$, Jung-Hee Bae ${ }^{2}$, Kyung-Seok $\mathrm{Hu}^{3}$, Hee-Jin $\mathrm{Kim}^{3}$ \\ ${ }^{1}$ Department of Dental Hygiene, Catholic Kwandong University, Gangneung, ${ }^{2}$ Department of Dental Hygiene, Division of Health Sciences, Namseoul \\ University, Cheonan, ${ }^{3}$ Division in Anatomy and Developmental Biology, Department of Oral Biology, Human Identification Research Center, BK21 \\ PLUS Project, Yonsei University College of Dentistry, Seoul, Korea
}

\begin{abstract}
The aims of this study were to clarify the topography and variations stylopharyngeus (STP) and superior constrictor (SC) muscles, and to examine what role they play in the pharyngeal movement. Forty-four specimens (22 right and 22 left sides) from embalmed Korean adult cadavers (13 males, 9 females; age range, 46-89 years; mean age, 69.2 years) were used in this study. The accessory bundle of STP and petropharyngeus was found in $18.2 \%(8 / 44)$ and $25.0 \%(11 / 44)$ of cases, respectively. A variation of the STP, in which it ran transversely and merged with the SC muscle, was found in 2.3\% (1/44) of cases while a variation of the SC muscle, in which it ran longitudinally and merged with the contralateral constrictors, was found in $11.4 \%$ (5/44). The variant muscle bundles play their own role in pharyngeal movement according to their morphology. These results provide information that will help a comprehensive understanding of the effects of pharyngeal muscles on movement.
\end{abstract}

Key words: Pharyngeal muscles, Stylopharyngeus, Velopharyngeal sphincter, Deglutition, Anatomy

Received June 22, 2020; Accepted July 7, 2020

\section{Introduction}

The pharynx, a tube-like structure, is a part of the digestive system and is composed of six muscles. It is well known that three external circular muscles (superior constrictor [SC], middle constrictor [MC], inferior constrictor [IC]) act as the sphincter, and three internal longitudinal muscles (the stylopharyngeus [STP], palatopharyngeus, and salpingopharyngeus) elevate the pharynx [1]. These muscles are not simple structures and do not work individually; they move and function together in sync. Therefore, a complete understand-

\footnotetext{
Corresponding author:

Hee-Jin Kim (iD

Department of Oral Biology, Yonsei University College of Dentistry, Seoul 03722, Korea

E-mail: hjk776@yuhs.ac
}

ing of the pharyngeal function requires a detailed knowledge on the morphology of each muscle and relationships between them [2].

Almost all muscles of the pharynx originate and insert into the internal surface of the pharynx. As an exception, the STP arises from the outside of the pharynx (i.e., the styloid process) and penetrates the posterior wall (i.e., the pharyngeal constrictor muscles) into the pharynx. Thus, there is a particularly close relationship between the STP and the SC muscle [3]. It is well known that the STP is critically involved in shortening the pharynx and elevating the larynx while the SC muscle plays a critical role as a sphincter muscle. However, the two muscles do not work independently. Their interaction can only be elucidated by observing their topographic relationship on both the inner and outer surfaces of the pharynx.

Previous studies have considered the functions of the

\section{Copyright ( 92020 . Anatomy \& Cell Biology}

This is an Open Access article distributed under the terms of the Creative Commons Attribution Non-Commercial License (http://creativecommons.org/licenses/by-nc/4.0/) which permits unrestricted non-commercial use, distribution, and reproduction in any medium, provided the original work is properly cited. 
SC muscle from the perspective of its anatomical morphology: it is attached to the buccinator muscle, which enables a smooth transition from the lingual stage to the pharyngeal stage during ingestion [4]. Moreover, some fibers of the SC muscle are directly attached to the velum, and thus, might assist in retraction thereof [5]. However, more detailed study is needed to understand the topographic characteristics and anatomical variations of the SC muscle.

The aims of this study were to clarify the topography and variations of both STP and SC muscles, to validate anatomical relationship between STP and SC, and to examine what role they play in the pharyngeal movement.

\section{Materials and Methods}

Forty-four specimens (22 right and 22 left sides) from 22 embalmed Korean adult cadavers (13 males, 9 females; age range, $46-89$ years; mean age, 69.2 years) were used in this study.

The pharynx was separated from the skull base, and the cervical vertebrae in the posterior wall was removed to allow for a clear observation of the pharyngeal posterior wall. The specimens included an origin of the STP with the styloid process.

The pharyngeal musculature was observed through careful dissection using the aid of a surgical microscope (OPMI-

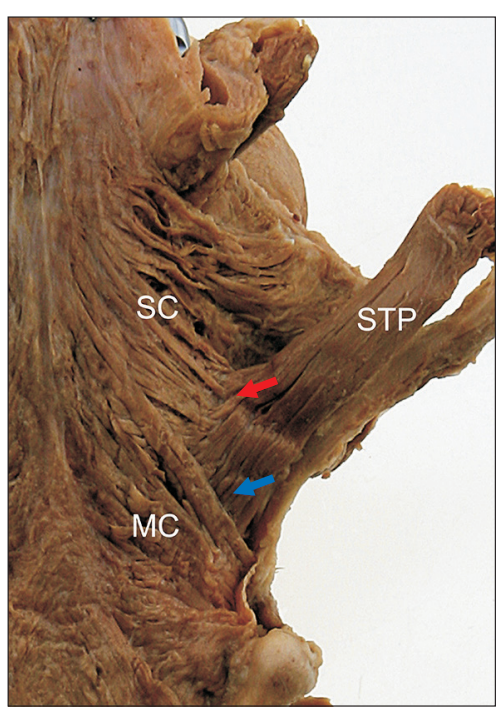

Fig. 1. General morphology of the STP muscle. At the insertion area, the upper fibers (red arrow) of the STP were intermingled with the SC muscle. The lower fibers (blue arrow) of the STP passed between the SC and the MC muscle. MC, middle constrictor; SC, superior constrictor; STP, stylopharyngeus.
FC; Carl Zeiss, Oberkochen, Germany). The neurovascular bundle, innervating the pharyngeal muscles, was removed from the posterior side of the pharynx to allow for a clear observation of the muscular morphology of pharyngeal muscles. The STP and three constrictor muscles were observed. Some variations in their topography along with additional bundles of STP fibers were classified according to origin and insertion site.

All photographs and diagrams in this article are structures viewed from the right side of the pharynx.

\section{Results}

In the posterior wall of the pharynx, the STP originated from the medial side of the styloid process and descended toward the lateral side of the pharynx. In the insertion area, the upper fibers of the STP were intermingled with the SC muscle while the lower fibers of the STP passed between the superior and MC muscles (Fig. 1). Lower fibers of the STP then attached to the posterior wall of the pharynx, together with the palatopharyngeus, on the inner surface.

An accessory bundle of the STP, which originated from the fascia around it, was found in 8 of the 44 specimens (18.2\%). This accessory bundle could be classified into two types, $\mathrm{A}$ and $\mathrm{B}$, depending on its insertion. Type $\mathrm{A}$, in which
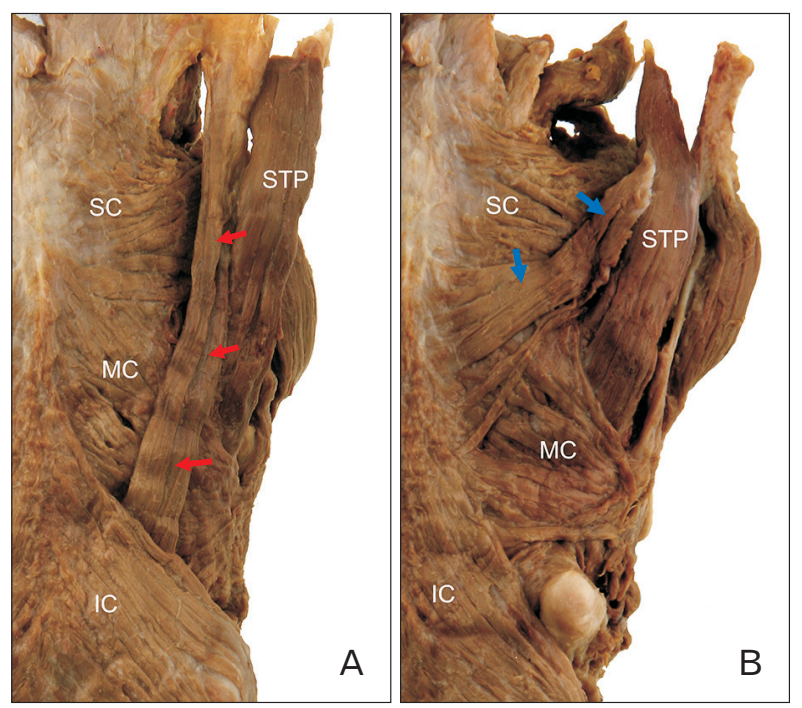

Fig. 2. Two types of accessory bundles of the STP muscle. (A) The accessory bundle of the STP (red arrows) passed between the MC and IC muscles. (B) The accessory bundle of the STP (blue arrows) inserted into the pharyngeal raphe on the midline with the SC muscle. IC, inferior constrictor; MC, middle constrictor; SC, superior constrictor; STP, stylopharyngeus. 
the accessory bundle of the STP passes between the middle and IC muscles, was observed in 9.1\% (4/44) of cases. Type B, in which the accessory bundle of the STP inserted into the pharyngeal raphe on the midline with the SC muscle, was observed in 9.1\% (4/44) of cases (Fig. 2).

The petropharyngeus, which is an occasional accessory levator muscle of the pharynx, was found in $25.0 \%$ of cases $(11 / 44)$. It arose from the undersurface of the petrous portion of the temporal bone and inserted into the pharynx. In most cases, this muscle was very thin, small, and merged into the SC muscle. A large petropharyngeus muscle was found in only one of the specimens, in which it divided two large bundles-both of which ran longitudinally. One of the bundles merged into the MC muscle, and the other passed deeply down the SC muscle (Fig. 3).

In only one specimen (2.3\%), the STP ran transversely and merged into the SC muscle. In this case, the STP contributed to the formation of the posterior wall such as the SC muscle (Fig. 4).

Variations of the constrictor muscles were found in the posterior wall of the pharynx in five cases (11.4\%). A part of the SC muscle ran longitudinally. In three of the five cases, the longitudinal fibers of the SC muscle passed between the superior and MC muscles with the STP. In the remaining two cases, these fibers merged with the MC muscle and con-
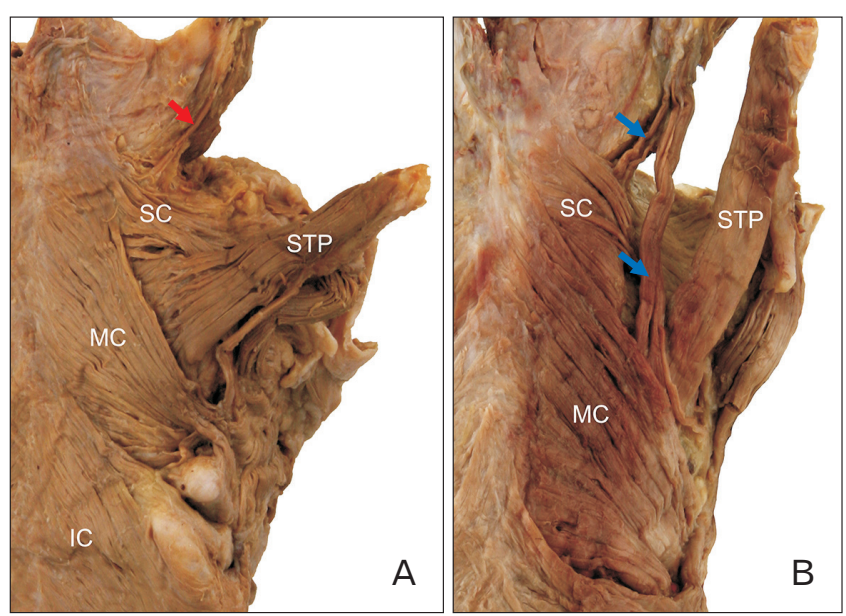

Fig. 3. Two types of the petropharyngeus muscle. The petropharyngeus muscle arose from the undersurface of the petrous portion of the temporal bone and inserted into the pharynx. (A) In most cases, these muscles had small and thin fibers (red arrow). (B) In one case, the muscle was large (blue arrows). It divided two bundles, one of which merged into the MC muscle while the other passed deeply down the SC muscle. IC, inferior constrictor; MC, middle constrictor; SC, superior constrictor; STP, stylopharyngeus. tralateral constrictor muscles (Fig. 5).

\section{Discussion}

This study observed the origin and variations, therein, of the STP with the SC muscle and considered its function based on its morphology. According to the present findings, the STP had accessory bundles in $18.2 \%$ (8/44) of cases. Ac-

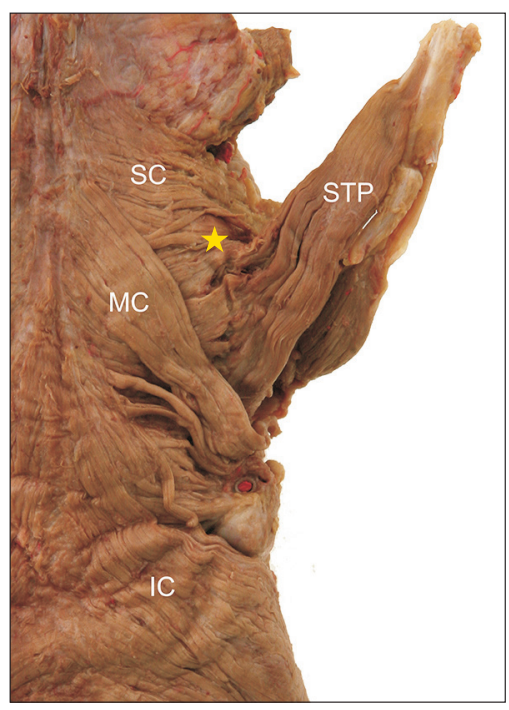

Fig. 4. Variation of the STP muscle. The STP ran transversely and merged into the SC muscle. Asterisk, area of the STP continuous with the SC; IC, inferior constrictor; MC, middle constrictor; SC, superior constrictor; STP, stylopharyngeus.
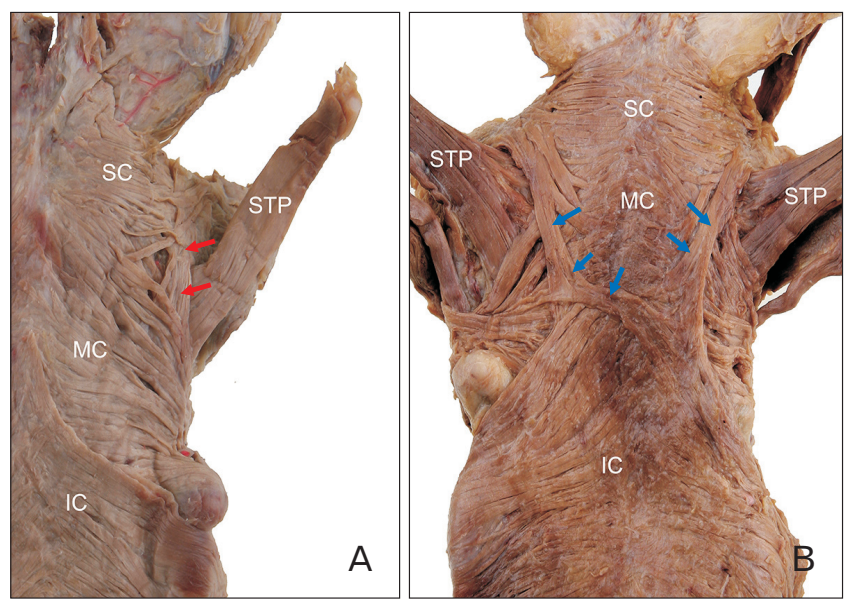

Fig. 5. (A) Some fibers of the SC (red arrows) ran longitudinally and passed between the SC and the MC muscle with the STP muscle. (B) Some fibers of SC (blue arrows) merged with the MC and contralateral constrictor muscles. IC, inferior constrictor muscle; MC, middle constrictor; SC, superior constrictor; STP, stylopharyngeus. 
cessory bundle was classified two type according to its running pattern. The STP with an accessory bundle had thinner than without an accessory bundle, so it may reduce its pharyngeal shortening function. An accessory bundle was thick as if it compensates for the thin STP. In type A, an accessory bundle ran longitudinally and may help the STP performing pharyngeal shortening. While type $\mathrm{B}$, an accessory bundle ran inferomedially and inserted into the pharyngeal raphe. It is not considered to work for only pharyngeal shortening with STP, but it might work to sphincter with SC. Whether the accessory bundle was running longitudinally or inferomedially, once accessory bundle was present, the function of STP may be reduce because STP was small muscle thickness.

In a specimen with another variation, it has thicker STP. However, even if STP was thick, it could not be considered to increase pharyngeal shortening function. A variant STP did not pass between the superior and MCs. It ran transversely and merged into the SC, and work with constrictor. This variant STP may play a role that was unknown until now.

As is well known, styloid process that is the origin area of STP is located behind of posterior wall of pharynx that is insertion area of it. Thus, this variant STP could pull posterior wall of pharynx posteriorly and causes an expanded anteroposterior diameter and reduced transverse diameter. In this study, only one specimen was found, further studies using more specimens are needed to assess its physiological and kinematics effects.

The petropharyngeus is a variation of the STP and was described in textbooks in various manners as inserting into the SC muscle [6], penetrating the SC muscle [7], or not even being described at all [8]. This variability of insertion and morphology has also been described [9]. The findings of the present study are consistent with these descriptions in that the petropharyngeus inserted into the outer surface of the superior or MC muscles or mingled with the outer surface fascia or IC muscle. Most of these small fibers descended or merged into the SC muscle.

The present study classified the petropharyngeus according to size. A petropharyngeus was observed in 11 of the 44 specimens (25.0\%). Shimada et al. [9] reported a prevalence of $1.4 \%$ among their specimens. The discrepancy between these findings may be attributed to the use of a surgical microscope in the present study and to the removal of all arteries and nerves during dissection, making it easier to observe small items such as these small muscle fibers. Although the prevalence of the petropharyngeus is quite high, it is difficult to assign a function to it since it is a small structure in all but one case.

The SC muscle was one of three constrictors in the pharynx. This muscle can be divided into four parts depending on its origin: pterygopharyngeal, buccopharyngeal, mylopharyngeal, and glossopharyngeal. The fibers curve backwards to insert into the median raphe $[1,4,10]$. In the present study, 5 of the 44 specimens exhibited a variation with respect to the SC muscle running pattern, in which a part of the muscle-fibers of the buccopharyngeal, in particularran longitudinally. Thispart appears to assist the longitudinal muscles in pharyngeal shortening. Some of the fibers merged with the MC muscle and contralateral constrictors. This pattern may help with harmonious deglutition movements among the three constrictor muscles.

In this study, it was found that two pharyngeal muscles, SPT and SC, have many anatomical variations. They show different running patterns and sizes, and each muscle bundle play their own role in pharyngeal movement according to their morphology. These results provide information that will help a comprehensive understanding of the effects of pharyngeal muscles on movement.

\section{ORCID}

Da-Yae Choi: https://orcid.org/0000-0003-2020-2665

Jung-Hee Bae: https://orcid.org/0000-0002-9058-3494

Kyung-Seok Hu: https://orcid.org/0000-0002-9048-3805

Hee-Jin Kim: https://orcid.org/0000-0002-1139-6261

\section{Author Contributions}

Conceptualization: KSH. Data acquisition: DYC, JHB. Data analysis or interpretation: DYC, JHB. Drafting of the manuscript: DYC. Critical revision of the manuscript: DYC, $\mathrm{HJK}$. Approval of the final version of the manuscript: all authors.

\section{Conflicts of Interest}

No potential conflict of interest relevant to this article was reported.

\section{References}

1. Standring S, Borley NR, Gray H. Gray's anatomy: the anatomi- 
cal basis of clinical practice. 40th ed. Edinburgh: Churchill Livingstone; 2008.

2. Choi DY, Bae JH, Youn KH, Kim HJ, Hu KS. Anatomical considerations of the longitudinal pharyngeal muscles in relation to their function on the internal surface of pharynx. Dysphagia 2014;29:722-30.

3. Meng H, Murakami G, Suzuki D, Miyamoto S. Anatomical variations in stylopharyngeus muscle insertions suggest interindividual and left/right differences in pharyngeal clearance function of elderly patients: a cadaveric study. Dysphagia 2008;23:251-7.

4. Tsumori N, Abe S, Agematsu H, Hashimoto M, Ide Y. Morphologic characteristics of the superior pharyngeal constrictor muscle in relation to the function during swallowing. Dysphagia 2007;22:122-9.

5. Perry JL. Anatomy and physiology of the velopharyngeal mechanism. Semin Speech Lang 2011;32:83-92.

6. Schünke M, Ross LM, Lamperti ED, Schulte E, Schumacher U, Rude J, Voll M, Wesker K. Thieme atlas of anatomy. New York: Thieme; 2006.

7. Moore KL, Dalley AF, Agur AMR. Clinically oriented anatomy. 6th ed. Philadelphia: Wolters Kluwer/Lippincott Williams \& Wilkins; 2010.

8. Tank PW, Gest TR, Burkel WE. Lippincott Williams \& Wilkins atlas of anatomy. Philadelphia: Wolters Kluwer Health/Lippincott Williams \& Wilkins; 2009.

9. Shimada K, Yokoi A, Ozawa H, Kitagawa T, Tezuka M. Observation of the petropharyngeal muscle in Japanese. Anat Anz 1991;173:193-8.

10. Kahrilas PJ. Pharyngeal structure and function. Dysphagia 1993;8:303-7. 